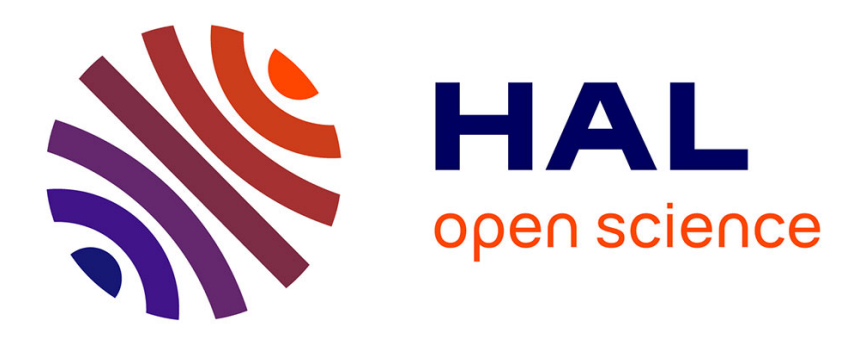

\title{
Soliton lattice melting-functional integral method
}

I. Batistić, S. Barišić

\section{To cite this version:}

I. Batistić, S. Barišić. Soliton lattice melting-functional integral method. Journal de Physique Lettres, 1979, 40 (23), pp.613-617. 10.1051/jphyslet:019790040023061300 . jpa-00231700

\section{HAL Id: jpa-00231700 https://hal.science/jpa-00231700}

Submitted on 1 Jan 1979

HAL is a multi-disciplinary open access archive for the deposit and dissemination of scientific research documents, whether they are published or not. The documents may come from teaching and research institutions in France or abroad, or from public or private research centers.
L'archive ouverte pluridisciplinaire HAL, est destinée au dépôt et à la diffusion de documents scientifiques de niveau recherche, publiés ou non, émanant des établissements d'enseignement et de recherche français ou étrangers, des laboratoires publics ou privés. 


\title{
Soliton lattice melting-functional integral method
}

\author{
I. Batistić and S. Barišić \\ Institute of Physics of the University, Zagreb, Croatia, Yugoslavia
}

(Reçu le 6 juin 1979, accepté le 16 octobre 1979)

\begin{abstract}
Résumé. - Nous déterminons les facteurs de structure pour la fonte à basse température du réseau de soliton présent dans le problème de la commensurabilité à une dimension, dans le cas d'épitaxie et d'öndes de densité de charge. Nous discutons brièvement de la relation de ce problème avec celui de l'infra-rouge dans l'approche sine-Gordon.
\end{abstract}

\begin{abstract}
Structure factors which describe the melting of the soliton lattice at low temperatures are found for the epitaxial and the CDW one-dimensional commensurability problem. The relation to the infra-red problem in the sine-Gordon approach is briefly discussed.
\end{abstract}

The problem of the thermodynamics of an epitaxial chain or the similar problem of the commensurateincommensurate crossover of the linear charge density wave (CDW), has been approached until now by two methods. The first approach proceeds by functional integration of the local free energy $[1,2,3,4]$

$$
\begin{aligned}
f(x)=\frac{T_{0}}{2}\left(\frac{\partial \varphi}{\partial x}\right)^{2}- & T_{\mu}\left(\frac{\partial \varphi}{\partial x}\right) \\
& +\frac{T_{\mathrm{p}}^{2}}{16 T_{0}}(1+\cos M \varphi) .
\end{aligned}
$$

The continuum approximation for the positional variable $x$ is usually used for $T_{\mathrm{p}} / T_{0}$ small enough [5]. The continuous one-dimensional problems are free of the ultra-violet singularities but exhibit in turn the infra-red singularities. In the CDW case the freeenergy (1) neglects further the CDW amplitude fluctuations since the amplitude has a fixed, finite value. This is a good approximation at low enough temperatures $[6,7]$ provided that $T_{\mathrm{p}}$ is not too large. And this is just the interesting temperature range since the infra-red singularities force the phase transition to occur at $T=0$.

The free energy associated with eq. (1) was found by the transfer matrix method in refs. $[5,8,9,10]$. The correlation functions

$$
K_{\kappa}(x)=\left\langle\mathrm{e}^{\mathrm{i} \kappa \varphi(x)} \mathrm{e}^{-\mathrm{i} \kappa \varphi(o)}\right\rangle
$$

and

$$
G(x)=\left\langle[\varphi(x)-\varphi(o)]^{2}\right\rangle
$$

were also determined analytically [3] or numerically [10] by this method, in certain limits with respect to the parameters involved in eq. (1).

The other, perhaps physically more transparent approach $[11,12,13]$ is based on the sine-Gordon equation, which results from the functional minimization of the Landau functional given by eq. (1). This approach is exact at $T=0$. The variable $\varphi$ is homogeneous in the ground state

$$
M \varphi_{0}=\pi(2 n+1),
$$

$(n=0, \pm 1, \ldots)$ for $4 q^{1 / 2}>\pi \mu\left(T_{\mathrm{p}}>\pi T_{\mu}\right)$, but for $4 q^{1 / 2}<\pi \mu$ it changes into the soliton lattice

$$
\begin{aligned}
\varphi_{0}(x) & =\frac{2}{M} a m\left[\tau_{\mathrm{p}} m^{-1 / 2} x \mid m\right] \\
& =\frac{2}{M} a m\left[\frac{T_{\mathrm{p}} M}{4 T_{0} m^{1 / 2}} x \mid m\right],
\end{aligned}
$$

where $a m$ is the elliptic integral (Jacobi) amplitude function [14]. This transition occurs due to the lifting of the soliton-antisoliton energetic degeneracy by the $T_{\mu}$ term of eq. (1). We have introduced here the reduced variables (involving $T$ for later convenience) $\tau_{\mathrm{p}}=M T_{\mathrm{p}} / 4 T_{0}, \mu=2 T_{\mu} / M T, q=T_{\mathrm{p}}^{2} / 4 M^{2} T^{2}$ and $m$ is determined via the elliptic integral $E(m)$ by $4 q^{1 / 2} E(m)=m^{1 / 2} \mu$. It was further possible to find [11, 12 , 13] the excitations of the soliton lattice by expanding the sine-Gordon equation in terms of small $\varphi-\varphi_{0}$. Those excitations belong to the optical and the acoustical branch. Knowing the excitations 
one can attempt to calculate in the usual way the correlation functions at finite $T$. Closer examination of this procedure shows however that due to the associated infra-red singularity the thermal amplitudes of the long acoustical waves are not small. Hence the large wave-length behaviors have to be calculated in a better approximation. It is expected in fact that the soliton lattice melts at any finite $T$ so that the linearized solutions cannot be meaningful for the wave-lengths much larger than the correlation length of the soliton lattice.

Our aim is to treat this problem analytically by the functional integral method, and then to establish the correspondence of our results with the results of the sine-Gordon approach. This will allow a better physical understanding of the role of the sine-Gordon infra-red singularities in the melting of the soliton lattice. Further, we shall emphasize the difference of the epitaxial and the CDW structure factors.

The eigenvalue problem associated to eq. (1) is

$$
\left[\left(\frac{\partial}{\partial z}-\mu\right)^{2}+a_{\mathrm{s}}-2 q \cos 2 z\right] \Psi_{\mathrm{s}}(z)=0 \text {. }
$$

It involves the non-hermitean operator since $\mu \frac{\partial}{\partial z}$ is anti-hermitean. Thus, beside the right eigenfunctions $\Psi_{\mathrm{s}}$ we also need the left eigenfunctions $\Phi_{\mathrm{s}}^{*}$ to form a biorthogonal basis. $\Phi_{s}^{*}$ satisfy eq. (5) with $\mu$ replaced by $-\mu$. In the manifold of states $\Psi_{\mathrm{s}}$ we choose those which satisfy Bloch's theorem

$$
\mathrm{e}^{\mathrm{i} \pi s} \Psi_{\mathrm{s}}=\Psi_{\mathrm{s}}(z+\pi),
$$

with $s$ real, since all potential wells are equivalent. We emphasize that it is necessary to take the continuum of states at the outset in order to get the correct infra-red behaviors of the correlation functions, and not only those which satisfy $\Psi_{s}(z+M \pi)=\Psi_{s}(z)$ of the quantum rotator. In fact the relevant states will be determined by the particular correlation function under consideration. The thermodynamic correlation function can be expressed in terms of the matrix elements

$$
\langle r|\mathcal{O}[\varphi(x)]| s\rangle=\int \mathrm{d} z \Phi_{\mathrm{r}}^{*}(z) \mathcal{O}\left(\frac{2 z}{M}\right) \Psi_{\mathrm{s}}(z)
$$

by $\left(\tau=T / T_{0}\right)$

$$
\begin{aligned}
\langle o \mid o\rangle & \left\langle\mathcal{O}(\varphi(x)) \mathcal{O}^{*}(\varphi(o))\right\rangle=\sum_{s}|\langle 0|\mathcal{O}| s\rangle|^{2} \times \\
& \times \exp \left[-\frac{M^{2}}{8} \tau\left(a_{\mathrm{s}}-a_{0}\right) x\right], \quad x>0 .
\end{aligned}
$$

According to the choice of $\mathcal{O}$ only a given set of states $\Psi_{\mathrm{s}}$ will be involved in eq. (8), due to the selection rules for the matrix elements (7).
The term linear in $\partial / \partial z$ can be removed from eq. (5) by the gauge transformation

$$
F(z)=\Psi_{\mathrm{s}} \mathrm{e}^{-\mu z} .
$$

$F(z)$ satisfies now eq. (5) without the $\partial / \partial z$ term, i.e. the Mathieu equation. Therefore $F(z)$ can be taken in the form [14]

$$
F_{v}(z)=\mathrm{e}^{\mathrm{i} v z} P_{v}(z),
$$

with $P_{v}(z+\pi)=P_{v}(z)$. Combining eqs. (9) and (10) and comparing to eq. (6) we find

$$
v=s+\mathrm{i} \mu \text {. }
$$

The analytical properties of the eigenvalues of the Mathieu equation are such that for a real $q$ there is no level crossing. For a fixed set of parameters $v$ thus runs over the states which are analytical continuations of the $\mu=0$ band states.

The lowest eigenvalue, which determines the free energy, corresponds therefore to $s=0$, i.e. to $v=\mathrm{i} \mu$, in full agreement with ref. [8]. This energy is a continuous function of $\mu$. It belongs to the first unstable region of the Mathieu equation [14] and shows a marked change in behavior for $q \gg 1$ and

$$
a(v=\mathrm{i} \mu)=a_{0} \simeq-2 q .
$$

This corresponds to $\pi \mu \simeq 4 q^{1 / 2}$ and to $q \gg 1$. According to the discussion related to eq. (4) this change of the regime reflects the soliton lattice formation, which proceeds without any phase transition. Indeed, there is no level crossing at $\pi \mu \simeq 4 q^{1 / 2}$ even between the highly excited states, as was already stated above.

We turn now to the calculation of the correlation functions which show more explicitly the effects of the soliton lattice formation. The static structure factor for the lattice with the parameter $a$, which is in epitaxy with the lattice of the parameter $b$, such that $M a \simeq L b$ ( $M$ and $L$ integer), is given by

$$
S(k)=\int \mathrm{d} x \mathrm{e}^{\mathrm{i} \frac{L b}{M a} k x} K_{\kappa}(x) .
$$

Here $\kappa=k b / 2 \pi M a$ and $K_{\kappa}$ is defined in eq. (2).

The analogous quantity $S_{\mathrm{CDW}}(k)$ for the CDW problem is obtained by inserting in eq. (12) $K_{1}(x)$ instead of $K_{x}(x)$ and by replacing $L b / M a$ in the exponent by unity [15]. This latter step is formal and amounts to a suitable redefinition of the wave-vector. On the other hand the difference in the $K$ functions involved in the two problems is essential. It is related to the fact that in the $\mathrm{CDW}$ case $\varphi$ is the phase, in contrast to the epitaxial problem where $\varphi$ is the linear atomic displacement. Noting that in the plane wave representation for $\Psi_{\mathrm{s}}$ 


$$
\left\langle s^{\prime}\left|\mathrm{e}^{\mathrm{i} \kappa \varphi}\right| s\right\rangle=\sum_{n} A_{n} \delta\left(s-s^{\prime}+\frac{2 \kappa}{M}-2 n\right), \quad n=0, \pm 1, \pm 2, \ldots
$$

we find

$$
S(k)=\sum_{n}\left|A_{n}\right|^{2} \frac{\frac{M^{2}}{4} \tau \operatorname{Re}\left(a_{2 n-\frac{2 \kappa}{M}}-a_{0}\right)}{\left[\frac{M^{2}}{8} \tau \operatorname{Re}\left(a_{2 n-\frac{2 \kappa}{M}}-a_{0}\right)\right]^{2}+\left[\frac{L b}{M a} k-\frac{M^{2}}{8} \tau \operatorname{Im}\left(a_{2 n-\frac{2 \kappa}{M}}-a_{0}\right)\right]^{2}}
$$

and

$$
S_{\mathrm{CDW}}(k)=\sum_{n}\left|A_{n}\right|^{2} \frac{\frac{M^{2}}{4} \tau \operatorname{Re}\left(a_{2 n-\frac{2}{M}}-a_{0}\right)}{\left[\frac{M^{2}}{8} \tau \operatorname{Re}\left(a_{2 n-\frac{2}{M}}-a_{0}\right)\right]^{2}+\left[k-\frac{M^{2}}{8} \tau \operatorname{Im}\left(a_{2 n-\frac{2}{M}}-a_{0}\right)\right]^{2}} .
$$

Only the quantum rotator states are involved in $S_{\mathrm{CDW}}$ in contrast to $S$, where the translational nature of $\varphi$ results in the appearence of small $k$ 's in the matrix elements (13), and the spectrum $a_{\mathrm{s}}$. Eq. (15) was also obtained in ref. [10] and further treated numerically.

The correlation function $G$ of eq. (3) is the same in both CDW and epitaxy cases. We use an appropriate extension of the usual oscillator strength sum rule to express, after some algebra, its Fourier transform as

$$
G(k)=\frac{\tau}{k^{2}}-\frac{2}{k^{2}}\left(\frac{\tau_{p}^{2}}{2 M}\right)^{2} \sum_{n \neq 0}\left|A_{n+1}-A_{n-1}\right|^{2} \frac{8}{M^{2} \tau\left(a_{2 n}-a_{0}\right)\left[k^{2}+\left[\frac{M^{2}}{8} \tau\left(a_{2 n}-a_{0}\right)\right]^{2}\right]} .
$$

Here the leading infra-red singularity $\left(k^{-2}\right)$ is extracted out.

Being interested in the low temperature properties we can employ the WKB method to calculate $\Psi_{\mathrm{s}}$ and $a_{\mathrm{s}}$. For $\pi \mu \gg 4 q^{1 / 2}$ (i.e. $a_{0} \ll-2 q$ ) the WKB states are obtained by a straight-forward integration over the whole range of $z$, since the relevant energies are far below the values of the potential. In this way

$$
\Psi_{\mathrm{s}}=\frac{1}{\sqrt{2 \pi}} \exp [i s F(z \mid m)], \quad m \simeq \frac{4 q}{\mu^{2}} \ll 1,
$$

with $F$ being the incomplete elliptic integral of the first kind [14].

$\Psi_{\mathrm{s}}$ is a fast converging expansion in terms of plane waves which renders the general expressions (14), (15) and (16) particularly suitable. Indeed if we expand eq. (17) in terms of $q / \mu^{2}$ small, we find

$$
\Psi_{s}(z)=\frac{\mathrm{e}^{\mathrm{i} z s}}{\sqrt{2 \pi}}\left[1-\frac{q}{2 \mu^{2}}(\cos 2 z+\text { is } \sin 2 z)+O\left(\frac{q^{2}}{\mu^{4}}\right)\right],
$$

so that the only nonvanishing $A_{n}$ 's are

$$
\begin{aligned}
A_{0} & =1 \\
A_{ \pm 1} & =\mp\left(s^{\prime}-s \pm 2\right) \frac{q}{4 \mu^{2}}
\end{aligned}
$$

with

$$
a_{\mathrm{s}}=2 q+(s+\mathrm{i} \mu)^{2} .
$$

Although it would be consistent to add the term proportional to $q^{2} / \mu^{4}$ in the last eq. (19) we shall omit it here, because it is not essential for our discussion. Inserting the results (19) into eqs. (14) and (15) it follows that

$$
\begin{aligned}
& S(k)=\frac{\kappa^{2} \tau}{\left[\frac{\kappa^{2} \tau}{2}\right]^{2}+k^{2}}+\left(\frac{q}{4 \mu^{2}}\right)^{2} \frac{4 \kappa^{2}}{M^{2}}\left\{\frac{\tau(M-\kappa)^{2}}{\left[\frac{\tau(M-\kappa)^{2}}{2}\right]^{2}+\left[k-\frac{\tau \mu M^{2}}{2}\right]^{2}}\right. \\
&\left.+\frac{\tau(M+\kappa)^{2}}{\left[\frac{\tau(M+\kappa)^{2}}{2}\right]^{2}+\left[k+\frac{\tau \mu M^{2}}{2}\right]^{2}}\right\},
\end{aligned}
$$




$$
\begin{aligned}
& S_{\mathrm{CDW}}(k)=\frac{\tau}{\left[\frac{\tau}{2}\right]^{2}+k^{2}}+\left(\frac{q}{4 \mu^{2}}\right)^{2} \frac{4}{M^{2}}\left\{\frac{\tau(M-1)^{2}}{\left[\frac{\tau(M-1)^{2}}{2}\right]^{2}+\left[k-\frac{\tau \mu M^{2}}{2}\right]^{2}}\right. \\
& \left.+\frac{\tau(M+1)^{2}}{\left[\frac{\tau(M+1)^{2}}{2}\right]^{2}+\left[k+\frac{\tau \mu M^{2}}{2}\right]^{2}}\right\}
\end{aligned}
$$

( $k$ is measured in (21) with respect to $2 k_{\mathrm{F}}$, where $k_{\mathrm{F}}$ is the Fermi wave-vector of the electron gas coupled to the lattice waves).

The correlation function $G$ is not directly measurable but it is most appropriate for the comparison with the results of the sine-Gordon approach $[11,12,13]$. From eq. (16) we find

$$
G(k)=\frac{\tau}{k^{2}}\left[1-2 \tau_{\mathbf{p}}^{4} \frac{k^{2}+\left(\frac{M^{2} \tau}{2}\right)^{2}-3 \mu^{2}\left(\frac{M^{2} \tau}{2}\right)^{2}}{\left(M^{2} \tau\right)^{2}\left(1+\mu^{2}\right)\left[\left(k+\frac{\tau \mu M^{2}}{2}\right)^{2}+\left(\frac{M^{2} \tau}{2}\right)^{2}\right]\left[\left(k-\frac{\tau \mu M^{2}}{2}\right)^{2}+\left(\frac{M^{2} \tau}{2}\right)^{2}\right]}\right]
$$

The leading terms in eqs. $(20,21,22)$ correspond to the well known results of the $d=1, n=2$ isotropic Landau model. The next order terms in these equations describe the effects related to the formation of the first harmonic $k=\tau \mu M^{2} / 2$ of the soliton lattice $\left(k=M\left(2 k_{\mathrm{F}}-g / M\right)\right.$ in the CDW case, $g$ reciprocal wave-vector). Viewed from the high-temperature side the soliton lattice forms primarily by the softening of the Fourier component which corresponds to its first harmonic. This is particularly clearly illustrated by eq. (22), which develops at $k=\tau \mu M^{2} / 2$ an acoustic like singularity at $T=0$, whereas at finite temperature the cut-off length is of the order $T_{0} / T$. It should however be remembered that we calculate only the static correlation functions so that nothing can be inferred about the dynamical features of the softening process. As for eq. (21), the leading term describes the homogeneous ordering of the phase over the length $\xi(T) \sim T_{0} / T$. The next order terms (the satellites) describe the appearence of the leading harmonics of the soliton lattice within the above domain. This superstructure is coherent over smaller distance of the order $\xi(T) /(M-1)^{2}$. The linearised collective excitations of the soliton lattice can probably be used in a meaningful way only within distances of the order $\xi(T)$ to $\xi(T) /(M-1)^{2}$. Note that in the $T=0$ limit the soliton lattice satellites in expressions (20) and (21) tend to the Bragg spots (planes).

We also notice from eq. (22) that the coefficient of the leading infrared singularity is affected by the soliton lattice term. Hence the large $x$ behaviour of its Fourier transform

$$
\left\langle[\varphi(x)-\varphi(o)]^{2}\right\rangle=Q|x|,
$$

although essentially unchanged with respect to that of the $d=1, n=2$ isotropic Landau model, has coefficient $Q$ somewhat modified.
It can be easily seen that each term in eqs. (14), (15) and (16) corresponds to a given (soft) soliton lattice harmonic. If the complete expression (17) for $\Psi_{\mathrm{s}}$ is used, all harmonics of the soliton lattice are retained. Instead of giving the corresponding lengthy expressions for the structure factors in reciprocal space, we prefer to illustrate this point by the $T=0$ limit of our expression in direct space

$$
K_{1}(x)=\mathrm{e}^{\mathrm{i} \varphi_{0}(x)}, \quad x>0,
$$

with $\varphi_{0}$ given by eq. (4).

The WKB treatment is not limited to

$$
a_{0} \ll-2 q\left(\pi \mu \gg 4 q^{1 / 2}\right) ;
$$

it can also be used in the opposite limit, provided that $T$ is small enough $(q \gg 1)$. Using the usual matching procedure (now $a_{0}>-2 q$ ) we have found the correlation functions in which, as expected, the soliton lattice reflections do not appear and the leading behaviour is that of the Ising model. However, the WKB procedure is not simply convergent for $a_{0} \simeq-2 q$, i.e. in the range of the order of

$$
|a-2 q|<\frac{\sqrt{q}}{12} \text { around } \pi \mu \simeq 4 q^{1 / 2} \text {. }
$$

Fortunately, the width of this range shrinks on lowering the temperature, in agreement with the result (24).

Acknowledgement. - We benefited from the discussions with A. Bjeliš, J. Friedel and E. Galleani d'Agliano. Part of the work was done when one of us (S.B.) was visiting at the Laboratoire de Physique des Solides, Orsay, France. 


\section{References}

[1] Frenkel, J. and Kontorova, T., Phys. Z. Sowjetunion 13 (1938).

[2] Frank, F. C. and Van Der Merwe, Proc. $R$. Soc. London 198 (1949) 205

[3] BrazovskiI, S. A., DzyaloshinskiI, I. E. and Obukhov, S. P., Zh. Eksp. Teor. Fiz. 72 (1977) 1550.

[4] McMillan, W. L., Phys. Rev. B 14 (1976) 1496.

[5] Guyer, R. A. and Miller, M. D., Phys. Rev. A 17 (1978) 1205.

[6] Uzelac, K. and Barišić, S., J. Physique Lett. 38 (1977) L-47.

[7] Batistić, I. and Barišić, S., submitted to Solid State Commun.

[8] Gupta, N. and Sutherland, B., Phys. Rev. A 14 (1976) 1790.

[9] GuYer, R. A. and Miller, M. D., Phys. Rev. Lett. 42 (1979) 718
[10] Oxwamoto, Y., Takayama, H. and Shiba, H., J. Phys. Soc. Japan 46 (1979) 1420. We are grateful to the referee for calling out attention to this work.

[11] BulaevsKII, L. N. and Khomski, D. I., Zh. Eksp. Teor. Fiz. 74 (1978) 1863.

[12] Pokrovski, V. L. and Talapov, A. L., Zh. Eksp. Teor. Fiz. 75 (1978) 1151.

[13] Theodorou, G. and Rice, T. M., Phys. Rev. B 18 (1978) 2840.

[14] Abramowitz, M. and Stegun, I. A., Handbook of mathematical functions (Dover, New York) 1970.

[15] BrazovskiI, S. A. and DzyaloshinsKiI, I. E., Zh. Eksp. Teor. Fiz. 71 (1976) 2338. 